\title{
Aspectos nutricionais de portadores de Síndrome de Down: Uma revisão bibliográfica
}

\author{
Nutritional aspects of down syndrome carriers: A bibliographic review
}

Aspectos nutricionales de portadores de síndrome de down: Una revisión bibliográfica

Débora Gomes de Sousa Araújo ${ }^{1}$, Larruama Priscylla Fernandes de Vasconcelos ${ }^{1 *}$, Erika Epaminondas de Sousa ${ }^{3}$, Paloma Cyntia da Silva Figueiredo Siqueira ${ }^{1}$, Rafael Ferreira Lima², Mayra Vieira Pereira Targino ${ }^{1}$, Luiza Satyro Morais de Medeiros ${ }^{1}$, Jullyane de Oliveira Maia Lemos ${ }^{3}$, Claudio Leite da Silva1, Raquel Bezerra de Sá de Sousa Nogueira ${ }^{1}$, Gabriela Miron de Sousa Vasconcelos ${ }^{1}$, Vanessa Bezerra Borges de Sousa ${ }^{1}$, Alcides Pereira Lino Júnior ${ }^{1}$.

\section{RESUMO}

Objetivo: Observar os aspectos nutricionais dos indivíduos acometidos por síndrome de Down (SD). Métodos: Tratou-se de uma revisão bibliográfica, de caráter descritivo e abordagem qualitativa dos dados, onde o processo de formulação se deu através de buscas por literaturas científicas, por meio de sites de iniciação científica dos últimos 10 anos, dando preferência as mais atuais. Resultados: Foram selecionados 22 artigos científicos, dados do ministério da saúde, 5 teses e 2 livros relativos ao tema, onde foi evidenciado os aspectos nutricionais de indivíduos com SD, as principais dificuldades/problemas destes indivíduos, onde sua maioria impacta diretamente no estado nutricional, assim como a importância do acompanhamento nutricional e da educação alimentar e nutricional, especialmente dos pais e/ou responsáveis, tendo em vista que o sobrepeso/obesidade é uma característica dominante nas pessoas acometidas pela referida síndrome, acentuando-se com a idade, apresentando na maioria das vezes dificuldades alimentares. Considerações finais: Concluiu-se que os indivíduos com SD apresentam muitos fatores que os predispõem a apresentarem sobrepeso/obesidade, como hipotireoidismo, hipotonia dos músculos, doenças cardiovasculares, taxa metabólica basal lenta, além dos hábitos alimentares inadequados e falta de atividade física, dessa forma, é de grande importância realizar acompanhamento nutricional com esses indivíduos desde os primeiros dias de vida, assim como trabalhar a educação nutricional, envolvendo todos que participam da vida da pessoa com SD.

Palavras Chaves: Aspectos Nutricionais, Consumo Alimentar, Síndrome Down.

\begin{abstract}
Objective: To observe the nutritional aspects of individuals with Down syndrome (DS). Methods: This was a bibliographical review, with a descriptive character and a qualitative approach to the data, where the formulation process took place through searches for scientific literature, through scientific initiation sites of the last 10 years, giving preference to the most current. Results: 22 scientific articles were selected, data from the Ministry of Health, 5 theses and 2 books related to the topic, where the nutritional aspects of individuals with DS were highlighted, the main difficulties / problems of these individuals, where most of them directly affect nutritional status, as well as the importance of nutritional monitoring and nutritional and nutritional education, especially of parents and / or caregivers, given that overweight / obesity is a dominant characteristic
\end{abstract}

\footnotetext{
${ }^{1}$ Faculdades Integradas de Patos (FIP).Patos-PB

2Universidade Federal de Campina Grande (UFCG). Pombal-PB

${ }^{3}$ Faculdade de Ciências Médicas de Campina Grande-PB. Campina Grande-PB

*E-mail: larruama priscylla@hotmail.com
}

SUBMETIDO EM: 10/2018

ACEITO EM: 11/2018

PUBLICADO EM: 01/2019

REAS/EJCH | Vol.Sup.21 | e562 | DOI: https://doi.org/10.25248/reas.e562.2019 Página 1 de 8 
in people affected by this syndrome, accentuating with age, presenting most often eating difficulties. Final considerations: It was concluded that individuals with DS have many factors that predispose them to being overweight / obese, such as hypothyroidism, muscle hypotonia, cardiovascular diseases, slow basal metabolic rate, as well as inadequate eating habits and lack of physical activity. of great importance to carry out nutritional monitoring with these individuals from the first days of life, as well as to work the nutritional education, involving all that participate in the life of the person with SD.

Key words: Nutritional Aspects, Food Consumption, Down syndrome

\section{RESUMEN}

Objetivo: Observar los aspectos nutricionales de los individuos acometidos por síndrome de Down (SD). Métodos: Se trató de una revisión bibliográfica, de carácter descriptivo y abordaje cualitativo de los datos, donde el proceso de formulación se dio a través de búsquedas por literaturas científicas, a través de sitios de iniciación científica de los últimos 10 años, dando preferencia a las más actuales.Resultados: Se seleccionaron 22 artículos científicos, datos del ministerio de salud, 5 tesis y 2 libros relativos al tema, donde se evidenciaron los aspectos nutricionales de individuos con SD, las principales dificultades / problemas de estos individuos, donde su mayoría impacta directamente en el estado nutricional , así como la importancia del acompañamiento nutricional y de la educación alimentaria y nutricional, especialmente de los padres y / o responsables, teniendo en cuenta que el sobrepeso / obesidad es una característica dominante en las personas acometidas por el referido síndrome, acentuándose con la edad, presentando en la mayoría de las veces dificultades alimentarias. Consideraciones finales: Se concluyó que los individuos con SD presentan muchos factores que los predisponen a presentar sobrepeso / obesidad, como hipotiroidismo, hipotonía de los músculos, enfermedades cardiovasculares, tasa metabólica basal lenta, además de los hábitos alimentarios inadecuados y falta de actividad física, , es de gran importancia realizar seguimiento nutricional con estos individuos desde los primeros días de vida, así como trabajar la educación nutricional, involucrando a todos los que participan en la vida de la persona con SD.

Palabras Claves: Aspectos Nutricionales, Consumo Alimentario, Síndrome Down.

\section{INTRODUÇÃO}

A síndrome de Down (SD) constitui-se uma condição geneticamente determinada, caracterizada pela presença extra de um cromossomo 21. A alteração genética na Síndrome de Down ocasiona problemas de ordem física e mental, isso faz com que os indivíduos com SD apresentem características especificas que os diferenciam dos indivíduos sem SD. Tal deficiência é responsável pelo atraso do desenvolvimento neuropsicomotor e por algumas características físicascomo face com perfil achatado, orelhas pequenas e arredondadas, excesso de pele na nuca, dentes pequenos, mãos curtas e largas, dedos curtos, pés curtos, largos e grossos (GALDINA AP, 2012; ALMEIDA MD et al., 2013).

Estes indivíduos exibem características metabólicas que os tornam mais suscetíveis ao surgimento de doenças relacionadas ao estado nutricional e que consequentemente afetam o equilibro do estado nutricional (GONÇALVES GF, 2013). Dentre tais características, que influenciam tanto a prática alimentar como o estado nutricional, pode-se citar: dificuldades de deglutição, língua protusa, retardo mental, alta suscetibilidade a infecções, taxa metabólica basal reduzida, hábitos alimentares inadequados, sedentarismo, alteração na glândula tireoide, compulsão alimentar, obstipação intestinal, hipotonia dos músculos (PIRES CLS, 2011; ROCHA RC, et al., 2015; CABRAL EMO e CORDEIRO FAM, 2017).

A obesidade é uma característica dominante nos indivíduos com Síndrome de Down, e a prevalência de sobrepeso e obesidade acentua-se com o avanço da idade como mostra o estudo de Bosco SMD et al. (2011) que teve como objetivo verificar a prevalência e sobrepeso e obesidade de pacientes portadores de SD nas 
escolas de Educação Especial do Vale do Taquari/RS. O número da amostra do estudo foi composta por 46 indivíduos portadores de SD com idades entre 2 e 50 anos. Os resultados encontraram que $56,5 \%$, da amostra apresentavam sobrepeso e obesidade, e verificaram que nos pacientes entre 2 e 18 anos, verificou-se eutrofia, e nos voluntários adultos com SD houve prevalência de sobrepeso e obesidade.

Outro estudo realizado por Rimmer JH et al. (2010) com 461 adolescentes americanos concluiu que os adolescentes com SD eram mais propensos a terem obesidade do que os adolescentes da população em geral. Esses resultados mostram que a manutenção do peso corporal é necessáriapara a prevenção de obesidade nessa população (ROIESEKI IM et al., 2010; FREIRE F et al., 2014). Silva ES et al. (2012) prioriza que a alimentação adequada e balanceada pode trazer benefícios para o bem estar, melhora no aprendizado e atenção, ajuda na manutenção da saúde e nutrição do indivíduo.

Indivíduos com SD apresentam ainda maiores chances de desenvolverem problemas da tireoide, que pode ser a causa da obesidade e sobrepeso, além de menor taxa metabólica basal, compulsão alimentar, hipotonia geral dos músculos, incluindo aqueles envolvidos na digestão. As alterações da tireoide ocorrem em 28 a $64 \%$ dos casos. A hipotonia muscular está presente em 100\% dos casos e tende a melhorar conforme 0 aumento da idade (CHAVES AL et al., 2008; MUSSOI TD, 2015; MARTINS NLM, 2015). A hipotonia pode dificultar os bons hábitos alimentares, devido a protrusão da língua, causada pela hipotonia dos lábios, causando problemas para mastigar, cuspir e reter o alimento (GONÇALVES GF, 2014).

Dessa forma, a avaliação nutricional torna-se imprescindível. Segundo Ribeiro CB e Toledo MT (2014), o objetivo de avaliar o estado nutricional é o de identificar distúrbios nutricionais como obesidade e sobrepeso dos portadores da SD, para que a partir do diagnostico se possa intervir de forma adequada, para assim recuperar e/ou manter o estado nutricional do indivíduo.

Dessa forma, o presente estudo objetivou observar os aspectos nutricionais dos indivíduos acometidos por SD, assim como a importância da educação alimentar e nutricional destes indivíduos e dos seus responsáveis, a fim de proporciona-los uma melhor qualidade de vida, tendo em vista que a SD traz consigo diversas dificuldades relativas ao perfil nutricional.

\section{MÉTODOS}

O presente estudo tratou-se de uma revisão bibliográfica, de caráter descritivo e abordagem qualitativa dos dados. O processo de formulação se deu através de buscas por literaturas científicas, por meio de sites de iniciação científica, como na Literatura Latino-americanas e do Caribe (LILACS), no banco de dados ScientificElectronic Library Online (SCIELO), na Literatura Latino-americanas e do Caribe (LILACS), no National Library of Medicina National (PUBMED), na base de dados do google acadêmico e em livros relacionados ao assunto, nas línguas portuguesa e inglesa, tendo como descritores: Aspectos Nutricionais. Consumo Alimentar e Síndrome Down.

Foram incluídos na pesquisa 34 artigos que apresentaram temática central do estudo dos últimos 10 anos (2003 a 2017), dando preferência as mais atuais e excluídos todos aqueles que não contribuiriam positivamente para o estudo e/ou não apresentaram a temática central da pesquisa, sendo utilizados artigos fora do período estipulado apenas quando necessário.

\section{RESULTADOS}

É visto a prevalência da obesidade em indivíduos acometidos pela Síndrome de Down. A associação entre obesidade e Síndrome de Down ganhou maiores dimensões por meio dos estudos de Chumlea WC e Cronk CE (1981) e Cronk C et al. (1988), onde eles avaliaram 262 crianças, com idade aproximada de 2 a 18 anos, de instituições em Melbourne, Wisconsin e de famílias de Boston, demonstrando que as crianças com Síndrome de Down apresentavam maiores valores de IMC do que as crianças do grupo controle.

Grande parte das pessoas com a síndrome de Down nascem com problemas cardiovasculares, o que pode contribui para o excesso de peso, por esse motivo a atividade física desses indivíduos tende a ser mais 
restrita. A ocorrência da doença cardíaca na SD é de 30 a 60\%, e quando não tratada aumenta-se o risco de mortalidade e de surgimento de hipertensão e insuficiência cardíaca, afetando a qualidade de vida destes indivíduos (BERTAPELLI F, 2012; GALDINA AP, 2012).

Outro problema encontrado são as doenças autoimunes que os predispõem a uma maior frequência de infecções recorrentes, como a doença celíaca e a alergia a proteína ao leite de vaca. Queiroz $\mathrm{F}$ et al. (2016) em sua pesquisa sobre o perfil nutricional de indivíduos com síndrome de Down, buscou verificar doenças relacionadas ao sistema imune na SD, sendo encontrado casos de alergia alimentar à proteína do leite de vaca. O estudo de Nisihara RM et al. (2005) mostrou alta prevalência 5,6\%de doença celíaca em crianças e adolescentes com síndrome de Down, perfazendo uma porcentagem de 5,6\% da amostra.

O zinco em quantidades baixas está relacionado as respostas imunológicas, poiso referido mineral é um co-fator de várias enzimas que participam do sistema imune. As alterações ocorridas na SD podem se agravar com a deficiência do zinco, que participa do metabolismo dos hormônios tri-iodotironina (T3) e tiroxina (T4). A baixa produção desses hormônios levam ao hipotireoidismo, ocasionando aumento de peso (GALDINA AP, 2012; SOUZA ACNM et al., 2013).

Alguns hábitos alimentares são frequentes nos portados de SD, como por exemplo, a recusa em comer alimentos que não são peneirados. Eles também têm dificuldade para mastigar, uma vez que sugam o alimento até ser amassado no céu da boca e engolido em seguida, além de apresentarem dificuldade de ingerir carne e alimentos de grupos específicos, como leite e vegetais, por exemplo. (GALDINA AP, 2012).

Muitos indivíduos com Síndrome de Down são alimentados pelos pais, mesmo possuindo capacidade para se alimentar sozinho. Algumas crianças mesmo chegando a maturidade para consumir alimentos mais sólidos continuam usando a mamadeira. Muitos recusam ingerir de alimentos crocantes, retem alimentos por longos períodos na boca, demoram a comer e tem dificuldade de usar utensílios (GALDINA AP, 2012).

De acordo com Roieski IM et al. (2010) indivíduos com síndrome de Down tem baixo consumo de hortaliças, frutas, leite e produtos lácteos, e um consumo exagerado de açúcares, cereais, pães, tubérculos e raízes. Essas preferências alimentares proporcionam um baixo consumo de fibras entre esses indivíduos.

A educação alimentar para os portadores da SD inclui; mastigar bem e várias vezes o alimento, colocar pequenas porções de comida na boca, fracionar as refeições e comer poucas quantidades de comida, comer com intervalos de 3 a 4 horas: café da manhã, lanche da manhã, almoço, lanche da tarde e jantar. Além de organizar uma dieta equilibrada, a qual todos os membros da família também possam aderiro tipo de alimento escolhido pela criança com SD, influenciando na educação alimentar (MOURA AB et al., 2009).

O consumo de doces, refrigerantes, bem como farináceos como massas devem ser evitado, ao passo que deve ser incentivado o consumo de frutas, legumes e verduras, especialmente frutas e/ou sucos ácidos, pois tornam a urina mais acida, diminuindo assim o surgimento de doenças como infecções urinarias, problema muito comum nos portadores de SD devido a hipotonia dos músculos (MOURA AB et al., 2009).

A avaliação do estado nutricional desses indivíduos é muito importante para que estes possam ter uma melhor qualidade de vida. A antropometria é um procedimento onde se mede a altura e o peso. É uma avaliação de baixo custo e aplicado globalmente, sendo o procedimento mais adequado para avaliar o estado nutricional. (GONÇALVES GF, 2013; QUEIROZ F et al., 2016).

Para avaliação antropométrica dos indivíduos com SD são recomendadas curvas próprias, uma vez que estes possuem déficit de crescimento e desenvolvimento e continuam ser pequenos na vida futura (QUEIROZ F et al., 2016).

As curvas de crescimento e peso mais utilizadas para a avaliação do estado nutricional são as curvas propostas por Cronk C et al. (1988), são curvas adaptadas para a Síndrome de Down de acordo com faixa etária e gênero. As medidas são mostradas em percentil de peso por idade (P/I), comprimento por idade (C/I) e estatura por idade (E/I) e indicam o estado nutricional desde o primeiro mês até os 18 anos de vida (MOURA AB et al., 2009; NUNES AM et al., 2016). 
A classificação é obtida, considerando baixo peso ou baixo para idade quando o percentil é menor que 5 (P5), eutrofia quando o percentil estiver entre 5 e 95 (P5- P95) e excesso de peso ou alto para idade, quando o percentil é maior que percentil 95 (P95) (PRADO ML et al., 2009).

Para maiores de 18 anos não há curvas específicas para avaliar o estado nutricional dos portadores da SD, estes devem ser avaliados conforme os pontos de corte estabelecidos pelo Sistema de Vigilância Alimentar e Nutricional (SISVAN- 2011).

O conhecimento sobre os hábitos alimentares não só se limita na prática clínica, mas é importante no campo da saúde pública com objetivo de orientação nutricional para prevenir e reduzir Doenças Crônicas Não Transmissíveis (DCNT) (MUSSOI TD, 2015).

Os distúrbios relacionados às práticas alimentares vistos na SD, relaciona-se as alterações anatômicasestruturais e hipotonias, tais distúrbios estão relacionadas ao surgimento de DCNT, tais como, diabetes, doenças cardiovasculares, hipertensão, problemas respiratórios, o que torna essencial conhecer o consumo alimentar para o diagnostico dessas condições para possível intervenção nutricional destes indivíduos (CHAVES AL et al., 2008; ROIESEKI IM et al., 2010).

Por meio da avaliação dietética é possível determinar se o hábito alimentar está adequado ou inadequado e os indicadores dietéticos são importantes para se determinar meios de intervenção nutricional (MUSSOI TD, 2015).

O Questionário de Frequência de Consumo Alimentar (QFA) é o método mais prático para avaliar o consumo alimentar e muito utilizado em estudos epidemiológicos. O QFA fornece informações sobre os hábitos alimentares de uma população, tendo como objetivo avaliar a frequência em que alimentos ou grupos de alimentos são consumidos durante certo período (ROIESEKI IM et al., 2014; MUSSOI TD, 2015).

Um estudo feito por Neves LF et al. (2015) demonstrou que a educação nutricional para pessoas com SD é uma estratégia adequada para promoção de saúde e mudança nos hábitos alimentares, pois estes indivíduos manifestam expressivas condições de aprendizado.

Giaretta A e Ghiorzi AR (2009) afirmam que as pessoas com SD possuem autonomia e independência relativas para escolher sua alimentação, com base em valores e crenças transmitidos pela família.

Dessa forma é necessário um trabalho educativo com todos que participam da vida do indivíduo com Síndrome de Down. Trabalho de conscientização e reeducação alimentar, no qual envolvam instituições de apoio a pessoa com SD, familiares, amigos e educadores (ROIESEKI IM et al., 2014).

\section{DISCUSSÃO}

A alimentação é um dos fatores que mais influência a saúde. Por meio dela consegue-se os nutrientes necessários para o bom funcionamento do organismo. Esta interfere no estado nutricional do indivíduo, onde hábitos alimentares inadequados estão associados ao surgimento das doenças crônicas não transmissíveis (DCNT) (SOUZA EA, et al., 2011; ZUCHETTO C, 2013; MARTINS NLM, 2015).

Uma alimentação adequada deve ter todos os grupos de alimentos, ser variada e colorida, esta pode prevenir e/ou tratar doenças, pois é rica em nutrientes que podem melhorar as defesas do organismo (MENDONÇA RT, 2010; FELIPPE F, et al., 2011).

Não há recomendações nutricionais específicas voltadas para os portadores de SD, o que se encontra na literatura são estudos que apresentam dados sobre curva de crescimento e desenvolvimento ponderoestatural (ALMEIDA MFA, et al., 2015).

A orientação dietética desses indivíduos são as mesmas preconizados para a população geral, onde para evitar possíveis deficiências ou excesso é necessário ofertar um aporte adequado de calorias e nutrientes (CHAVES AL, et al., 2008). 
O processo de nutrição adequada para indivíduo com SD precisa acontecer após o nascimento com o leite materno, pois os bebês apresentam dificuldades com a deglutição, o leite materno é o alimento mais completo nesta fase da vida e o bebê ao sugar o leite da mãe fortalecerá a musculatura da boca (ALMEIDA MFA, et al., 2015).

Muitos pais mantêm, por muito tempo na alimentação desses indivíduos, alimentos de fácil mastigação e deglutição, como líquidos e alimentos pastosos. Pois devido ao retardo mental da criança, fica mais complicado reconhecer quando ela está pronta para uma mudança na prática alimentar e os pais evitam uma progressão alimentar, o que torna-se mais difícil o controle deste hábito (ROIESEKI IM, et al., 2010).

Esse tipo de dieta é uma resposta emocional dos pais, que inconsciente querem superproteger e recompensar os filhos por terem a SD. Estes oferecem maior quantidade de comida do que o necessário, incluindo aqueles com alto teor de gordura e carboidratos simples, o que favorece o de desenvolvimento de sobrepeso e obesidade (ROIESEKI IM, et al., 2010).

Para se ter o bom funcionamento do intestino, é importante o consumo de frutas, legumes e verduras, uma vez esses alimentos contem fibras que não são digeridas e auxiliam na formação do bolo fecal (GALDINA AP, 2012).

Para avaliar o consumo alimentar vários instrumentos podem ser utilizados, e entre eles estão o recordatório de $24 \mathrm{~h}$, questionário de frequência alimentar e o registro ou diário alimentar. Não existe um método ideal, um parâmetro isolado não pode ser usado como indicador do estado nutricional (GUERRA I, 2004; MUSSOI TD, 2015).

Assim a decisão sobre qual o "melhor" método a ser utilizado deve considerar o objetivo do estudo, a população a ser estudada, os recursos disponíveis e a reprodutibilidade do método, além de outros fatores como como o número de pessoas investigadas e de entrevistadores disponíveis, a capacidade de compreensão dos indivíduos avaliados e o tempo para a coleta dos dados dietéticos (GUERRA, 2004; MUSSOI TD, 2015).

É de extrema importância desde os primeiros dias de vida o acompanhamento de um profissional nutricionista nos portadores de SD, por apresentarem alterações no seu estado nutricional, que vem desde o nascimento até a fase adulta (MOURA AB, et al., 2009).

A obesidade e outras doenças associadas à SD podem ser prevenidas com acompanhamento nutricional, pois hábitos nutricionais definidos por erros dietéticos são os principais fatores do desenvolvimento de obesidade nesta população (PRADO ML, et al., 2009).

A participação dos pais nas atividades de educação nutricional é de suma importância para que os objetivos sejam alcançados, pois a família é o meio externo que mais tem influência na alimentação, pois é no ambiente familiar onde se formam os hábitos alimentares (GIARETTA A e CHIROZI AR, 2009; ROIESEKI IM, et al., 2010).

O nutricionista pode promover a educação nutricional com a família, orientando sobre quantidade e qualidade apropriadas das refeições. Ajudando as famílias a estabelecer um plano de refeições e auxiliar a identificar formas de alterar o comportamento alimentar negativo do indivíduo com SD (ZUCHETTO C, 2013).

\section{CONSIDERAÇÕES FINAIS}

Com este estudo pode-se concluir que os indivíduos com Síndrome de Down têm muitos fatores que os predispõem a terem sobrepeso e obesidade, como hipotireoidismo, hipotonia dos músculos, doenças cardiovasculares, taxa metabólica basal lenta, além dos hábitos alimentares inadequados e falta de atividade física.

Dessa forma, percebe-se que acompanhamento nutricional desde os primeiros dias de vida é de grande importância, assim como a educação nutricional, e que esta seja trabalhada com todos os envolvidos na vida 
da pessoa com Síndrome de Down, garantindo assim a escolha de bons hábitos alimentares e melhor qualidade de vida.Ressalta-se que o nutricionista deve esta embasado sobre o assunto, para que possa da melhor suporte nutricional para a família.

Sugere-se a criação de curvas específicas para adultos com Síndrome de Down, pois estes são avaliados pelo IMC, podendo ocorrer erros de diagnóstico nutricional, e a criação de recomendações nutricionais específicas. Dessa forma é necessário que novos estudos sejam realizados no desenvolvimento de novas referências para contribuir com crescimento dessa área.

\section{REFERÊNCIAS}

1. ALMEIDA MD, MOREIRA MCS, TEMPSKI PZ. A intervenção fisioterapêutica no ambulatório de cuidado a pessoa com síndrome de Down no Instituto de Medicina Física e Reabilitação HC FMUSP. Acta Fisiatr., 2013;20(1):55-62.

2. ALMEIDA MFA, OLIVEIRA AC, SCHITKOSKI, MC et al. Educação nutricional e qualidade de vida para portadores de Síndrome de Down (SD). FiepBulletin - Online, 2015; 85(1):284-289.

3. BERTAPELLI F. Composição corporal e somatótipo em pessoas com Síndrome de Down [Tese]. Campinas: Faculdade de Educação Física Unicamp: Faculdade de Educação Física Unicamp; 2012.

4. BOSCO SMD, SCHERER F, ALTEVOGT CG. Estado nutricional de portadores de Síndrome de Down no Vale do Taquari. conSientiae saúde, 2011; 10(2):278-284.

5. CABRAL EMO, CORDEIRO FAM. Avaliação antropométrica e consumo alimentar de crianças portadoras de Síndrome de Down acompanha das pela ASPAD do município de Jacareí, SP. Revista Científica UMC, 2017; 2(1):1-11.

6. CHAVES AL, CAMPOS CK, NAVARRO AC. Relação Da Síndrome De Down Com A Obesidade. Rev. Bras. de Obesidade, Nutrição e Emagrecimento,. 2008;2(11):412-422.

7. CHUMLEA WC, CRONK CE. Overweight among children with trisomy 21. Journal of Intellectual Disability Research. 1981; 25(4): 275-280.

8. CRONK C, CROCKER AC, PUESCHEL SM et al. Growth charts for children with Down syndrome: 1 month to 18 years of age. Pediatrics, 1988; 81(1):102-110

9. FELIPPE F, BALESTRIN L, SILVA FM et al. Qualidade da dieta de indivíduos expostos e não expostos a um programa de reeducação alimentar. Rev. Nutr., 2011; 6(24):833-844.

10. FREIRE F, COSTA LT, GORLA JI. Indicadores de obesidade em jovens com síndrome de down. Motrocidade. $2014 ; 10(2): 2-10$.

11. GALDINA AP. A importância do cuidado nutricional na Síndrome de Down.[Monografia]. Criciúma: Universidade do Extremo Sul Catarinense- Unesc; 2012.

12. GIARETTA A, CHIORZI AR. O ato de comer e as pessoas com Síndrome de Down. Revista Brasileira de Enfermagem, 2009; 62(3):480-484.

13. GONÇALVES GF. Avaliação nutricional dos alunos da apae de vila velha, com síndrome de down, paralisia cerebral e/ou autismo. [Monografia]. Vitória: Faculdade Católica Selesiana do Espírito Santo; 2013.

14. GUERRA I. Importância da alimentação e da hidratação do atleta. Min. Educ. Fís., 2004; 12(2):159-173.

15. MARTINS NLM. Intervenção mediacional e hábitos alimentares: Um estudo de crianças com síndrome de Down. [Dissertação]. Uberlândia: Universidade Federal de Uberlândia; 2015.

16. MENDONÇA RT. Nutrição um guia completo de alimentação, práticas de higiene, cardápios, doenças, dietas e gestão. São Paulo: Rideel; 2010.

17. MOURA AB, MENDES A, PERI A et al. Aspectos nutricionais em portadores da Síndrome de Down. Cadernos da escola de saúde, 2009; 2(1):1-11.

18. MUSSOI TD. Avaliação nutricional da prática clínica: da gestação ao envelhecimento. 1 ed. Rio de Janeiro: Guanabara Konogan; 2015.

19. NEVES LF, DURÕES GLLS, BEZERRA KFet al. Hábitos alimentares: sua influência no índice de massa corporal (IMC) em portadores de Síndrome de Down. Caderno de Ciências Agrárias, 2015; 7(2):40-44.

20. NISIHARA RM, KOTZE LM, UTIYAMA SR. et al. Doença celíaca em crianças e adolescentes com síndrome de Down. J. Pediatr., 2005; 81(5):373-376.

21. NUNES AM, SOUSA AMA, SÁ OMS et al. Diagnóstico nutricional de crianças e adolescentes com síndrome de down em Teresina - PI. Interd., 2016; 9(4):20-27. 
22. PRADO ML, MESTRINHERI L, FRANGELLA VS et al. Acompanhamento nutricional de pacientes com Síndrome de Down atendidos em um consultório pediátrico. O mundo da saúde, 2009; 33(3):335-346.

23. PIRES CLS. Perfil nutricional de crianças e adolescentes com síndrome de down. [Trabalho de Conclusão de Curso]. Guarapuava: Universidade Estadual do Centro Oeste; 2011.

24. QUEIROZ F, DE SANTADA MAC, SILVA MGV et al. Perfil nutricional de portadores de síndrome de down no agreste de Pernambuco. Nutr. Clín. Diet., 2016; 3(36):122-129.

25. RIBEIRO CB, TOLEDO MT. Avaliação antropométrica dos alunos portadores de síndrome de down da Apae de Araras-SP. O mundo Saúde, 2014; 3(38):334-340.

26. RIMMER JH, YAMAKI K, LOWRY BM, WANG E, VOGEL LC. Obesity and obesity-related secondary conditions in adolescents with intellectual/developmental disabilities. J Intellect Dis Res., 2010; 54(9):787-94.

27. ROCHA RC, LACERDA KC, DE MELO MM, et al. Avaliação antropométrica e consumo alimentar de crianças e adolescentes com Síndrome de Down de Ubá-Mg: Subsídio para a promoção da alimentação saudável. Rev. APS. 2010; 18(2): 151 - 156.

28. ROIESEKI IM, SILVA IM, CARDOSO FB et al. Avaliação Da Dieta Habitual De Adolescentes Com Síndrome De Down. Rev.Saúde.Com, 2010. 2010; 2(6):130-138.

29. ROIESKI IM, SILVA IL, REZENDE AAB et al. Avaliação do perfil nutricional de adolescentes com Síndrome de Down: Pressuposto epistemológico para um aconselhamento nutricional com enfoque na ergomotricidade. Rev. Amazônia Science\& Health, 2014; 2(2):21 - 28.

30. SILVA ES, COSTA NS, PEREIRA NMG et al. Importância da alimentação saudável para a promoção da qualidade de vida de indivíduos com síndrome de down. Rev. em foco, 2012; 1(23):84-93.

31. MINISTÉRIO DA SAUDE (BR). Orientações para a coleta e análise de dados antropométricos em serviços de saúde: Norma Técnica do Sistema de Vigilância Alimentar e Nutricional - SISVAN / Ministério da Saúde, Secretaria de Atenção à Saúde, Departamento de Atenção Básica. [Internet]. Secretaria de Atenção à Saúde. Departamento de atenção Básica. 2011. 76 p

32. SOUZA ACNM, RODRIGUES MC, FERREIRA LG. Excesso de peso e gordura corporal em portadores de síndrome de Down de uma instituição no município de Divinópolis-MG. Rev. Bras. de Ciências da Saúde, 2013; 37(1):31-39.

33. SOUZA EA, FILHO VCB, NOGUEIRA JAD et al. Atividade física e alimentação saudável em escolares brasileiros: revisão de programas de intervenção. Cad. Saúde Pública, 2011; 8(27):1459-1471.

34. ZUCHETTO C. Estado nutricional, consumo alimentar e atividade física de crianças e adolescentes com Síndrome de Down [Dissertação]. Pelotas: Universidade Federal de Pelotas: Universidade de Pelotas; 2013. 